tionally inelastic cross sections now readily calculable. The most exciting result, however, to appear recently, must be the ab initio R-matrix calculation of B.I. Schneider, M. Le Dourneuf and Vo Ky Lon on electron-nitrogen scattering near the $2.4 \mathrm{eV}$ resonance. By including the electronicnuclear interaction, they were able to reproduce faithfully all the vibrational substructure observed in the collisional cross sections near and at this resonance.

\section{An Acknowledgement}

In preparing this article, it was inevitable that a number of notable contributions to the subject of electron-molecule scattering would be omitted. For example, many significant insights into the nature of electron-molecule resonances have been afforded by the resonance theory of $\mathrm{N}$. Bardsley, A. Herzenberg, F. Mandl and others, and much has been contributed by L.A. Collin, M.A. Morrison, D.W. Robb and others to the computationally difficult problem of the inclusion of exchange in the numerical approaches. Similarly, many of my colleagues have put much effort into the very daunting question of how best to represent molecular polarisation in low energy electron scattering. These unavoidable omissions, however, only further

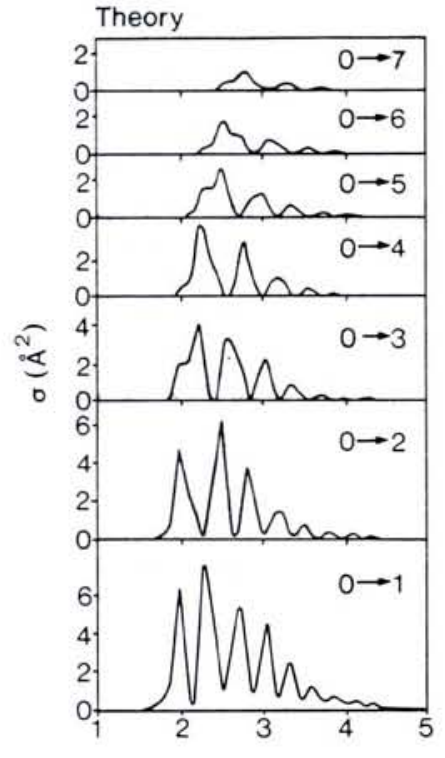

(eV)

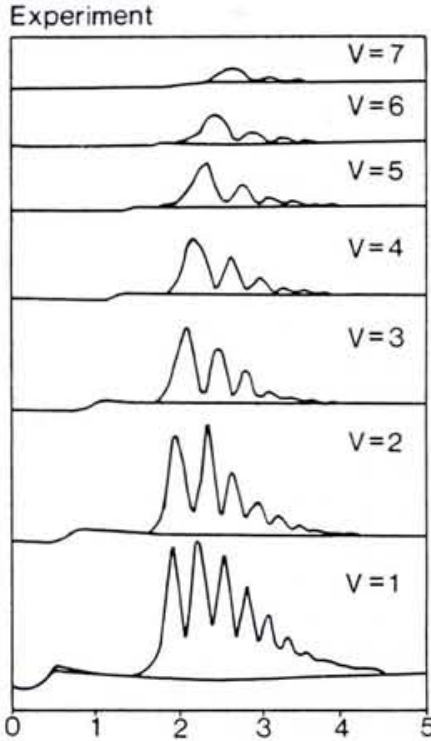

(eV)

Fig. 8 - An R-Matrix calculation of the vibrational excitation cross sections in $e^{-}+N_{2}$ near the $2.4 \mathrm{eV}^{2} \Pi_{g}$ resonance, (by B.I. Schneider, M. Le Dourneuf and Vo Ky Lan).

strengthen my assertion that atomic and molecular physics, and electron-molecule scattering in particular, are both important and exciting areas for a theorist to be working in today.

\section{Further Reading}

"Electron Molecule and Photon Molecule Collisions" Ed: T. Rescigno, V. McKoy, and B. Schneider. (Plenum Press, New York) 1979.

\title{
On-line Information Retrieval
}

Sir,

In your recent article "Searching the Literature: an enquiry by Europhysics News" (May 1980), you mention that Europhysics News knows of no single document which summarizes in any comprehensive way the services offered by the various hosts, and which include the data bases that are available and the sources from which these have been compiled.

I think you will find that HALL, J.L. Online Bibliographic Data bases 1979 Directory (Aslib: London, 1979) to some extent serves as such a document. Although mainly relating to on-line bibliographic data bases, this work also lists other related online data bases e.g. data banks and data bases planned to go on-line in the future. This directory could be supplemented by TOMBERG, Alex Data Bases in Europe: a directory to machine-readable data bases and data banks in Europe, 3rd. ed. (Aslib; London, 1977). Whilst both of these works are somewhat out of date, I understand that the work by Hall will be updated some time in the future. This indeed points to one of the major problems of producing such a directory - the field is constantly changing and any directory is likely to be out of date as soon as it is in print.

I would also like to point out a major advantage of the on-line systems that was not evident from your article. This relates to the fact that the on-line database allows one to search for the information in a fashion that is not available in the traditional abstracting or indexing journals. The traditional abstracting journals may be used in two basic ways:

(1) as a current awareness journal, i.e. the researcher turns to the relevant section of the journal and scans the entries covering his own field of interest or

(2) as a retrospective search aid, i.e. the researcher looks up the subject index for the topic he is interested in and this refers him to entries relating to that subject. The trouble with this retrospective search method is that the researcher is dependent on the indexing language used by the abs- tracting journal and this can be slow to pick up new terminology. This, in my opinion, is where one of the advantages of the online search technique comes in. As well as having this "controlled index" approach available to it (in most data bases e.g. INSPEC) it also has the facility to look for "key words" in the title, abstract or indexing terminology. Thus the researcher can often pick up references that he was unable to obtain using the conventional subject index.

\section{N. B. Butterwick}

Physics and Mathematical Sciences Librarian Queen's University, Belfast

\section{University of Neuchâtel Professorship in Solid State Physics}

Applications are invited for a full Professorship in Solid State Physics at the University of Neuchatel (Switzerland). The appointee is expected to stimulate and supervise research within a group of collaborators using existing equipment, which includes a van de Graaff accelerator for $3 \mathrm{MeV}$ protons, facilities for NMR, ESR and optical spectroscopy as well as for crystal growth, photolithography and cryogenics. He will participate in the teaching activities of the department in general physics and solid state physics. For further information, contact:

Prof. J. Rossel, Institut de physique, rue A.-L. Breguet 1, CH-2000 Neuchâtel. Letters of application, with a curriculum vitae and a list of publications, should be addressed to the:

Département de I'Instruction publique, Château, CH-2001 Neuchâtel, before $\mathbf{3 0}$ November, 1980. 